\title{
Heritability and genetic correlations of insulin resistance and component phenotypes in Asian Indian families using a multivariate analysis
}

\author{
D. Zabaneh • J. C. Chambers • P. Elliott • J. Scott • \\ D. J. Balding • J. S. Kooner
}

Received: 20 March 2009 / Accepted: 3 August 2009/Published online: 10 September 2009

(C) Springer-Verlag 2009

\begin{abstract}
Aims/hypothesis Insulin resistance and related metabolic disturbances are more common among Asian Indians than European whites. Little is known about the heritability of insulin resistance traits in Asian Indians. Our objective was to estimate heritabilities and genetic correlations in Asian Indian families. Methods Phenotypic data were assembled for 181 UK Asian Indian probands with premature CHD, and their 1,454 first-, second- and third-degree relatives. We calculated (narrow-sense) heritabilities and genetic correlations
\end{abstract}

Electronic supplementary material The online version of this article (doi:10.1007/s00125-009-1504-7) contains supplementary material, which is available to authorized users.

D. Zabaneh $(\bowtie) \cdot$ J. C. Chambers $\cdot$ P. Elliott $\cdot$ D. J. Balding

Department of Epidemiology and Public Health,

Imperial College London,

St Mary's Campus, Norfolk Place,

London W2 1PG, UK

e-mail: d.zabaneh@imperial.ac.uk

J. S. Kooner $(\bowtie)$

National Heart and Lung Institute, Imperial College London,

Hammersmith Hospital Campus,

Du Cane Road,

London W12 0NN, UK

e-mail: j.kooner@imperial.ac.uk

\section{J. Scott}

National Heart and Lung Institute, Imperial College London,

South Kensington Campus,

London, UK

Present Address:

D. Zabaneh

UCL Genetics Institute (UGI), Kathleen Lonsdale Building,

University College London,

Gower Street,

London WC1E 6BT, UK

e-mail: d.zabaneh@ucl.ac.uk for insulin resistance traits, and common environmental effects using all study participants and a multivariate model. The analysis was repeated in a subsample consisting of individuals not on drug therapy.

Results Heritability estimates (SE) for individuals not on drug therapy were: BMI 0.31 (0.04), WHR 0.27 (0.04), systolic BP 0.29 (0.03), triacylglycerol $0.40(0.04)$, HDLcholesterol 0.53 (0.04), glucose 0.37 (0.03), HOMA of insulin resistance (HOMA-IR) $0.22(0.04)$, and $\mathrm{HbA}_{1 \mathrm{c}} 0.60$ (0.04). We observed many significant genetic correlations between the traits, in particular between HOMA-IR and BMI. Heritability estimates were lower for all phenotypes when analysed among all participants.

Conclusions/interpretation Genetic factors contribute to a significant proportion of the total variance in insulin resistance and related metabolic disturbances in Asian Indian CHD families.

Keywords Asian Indian · Genetic correlations · Heritability · Insulin resistance $\cdot$ Multivariate analysis $\cdot$ Shared environment

\begin{tabular}{ll}
\multicolumn{2}{l}{ Abbreviations } \\
DBP & Diastolic BP \\
HOMA-IR & HOMA of insulin resistance \\
IR & Insulin resistance \\
SBP & Systolic BP \\
TG & Triacylglycerol
\end{tabular}

\section{Introduction}

Insulin resistance (IR), type 2 diabetes and associated disorders of dyslipidaemia, hypertension, coagulation abnor- 
malities and atherosclerosis [1] are major causes of morbidity and mortality, and are rapidly increasing globally [2]. Asian Indians (people from India, Pakistan, Bangladesh or Sri Lanka) have a high prevalence of IR and related metabolic disturbances compared with other populations. Among Asian Indians, IR and associated metabolic phenotypes are reported to account for up to $70 \%$ of the excess CHD risk, compared with European whites. The contribution of genetic factors underlying IR and its related metabolic disturbances amongst Asian Indians has not been extensively investigated. We therefore estimated the heritabilities and genetic correlations of IR and its component phenotypes using a multivariate model, in a large, well-characterised set of extended families of Asian Indian CHD patients.

\section{Methods}

Study population We identified 181 Asian Indian men and women with premature CHD, defined as myocardial infarction, coronary revascularisation or coronary artery stenosis $\geq 70 \%$ in one or more major epicardial vessel before the age of 60 years. All had been living in the UK for an average of 26 years (range 1-55 years), and originated predominantly from the Punjab state, India. Some were born overseas and some in the UK: 19\% born in Africa, $44 \%$ born in India, 35\% born in the UK and 2\% born in other countries. Recruitment was through Ealing and Hammersmith hospitals. We invited all available first-, second- and third-degree and spouse relatives of the 181 probands for phenotypic characterisation. In total 1,663 people were investigated, including the probands. From these individuals, 29 were excluded as having white European-descent ethnicity; this comprised any individual and his/her offspring. The remaining 1,634 individuals from 180 families were $86 \%$ Sikh and 14\% Hindu, a comparison between the two groups is in Electronic supplementary material (ESM) Table 1.

Clinical measurements Data were collected by trained nurses according to a standard protocol. Participants attended after an $8 \mathrm{~h}$ fast. Physical assessment included BP and anthropometric measurements (height, weight and waist and hip circumference), from which BMI (a measure of general adiposity) and WHR (a measure of central adiposity) were calculated. Fasting glucose, insulin, total and HDL-cholesterol and triacylglycerol (TG) were measured, and HOMA of insulin resistance (HOMA-IR) derived using fasting insulin and glucose concentrations [3]. Type 2 diabetes was defined as a physician diagnosis of diabetes, or blood glucose $\geq 7.0 \mathrm{mmol} / 1$ and hypertension as a physician diagnosis of hypertension on treatment, or BP $\geq 140 / 90 \mathrm{mmHg}$. The study was approved by the Local
Research Ethics Committee, and consent was obtained from all participants.

Statistical analysis Fasting glucose, HDL-cholesterol, TG and HOMA-IR were $\log$ transformed $\left(\log _{e}\right)$ to improve normality. All traits were adjusted for age and sex, and adjusted phenotypic values $>4$ SD below or above the mean were excluded. For heritability and genetic correlation estimation, the age- and sex-adjusted residuals were analysed using VCE version 6 , which provides heritability estimates under an additive genetic model [4]. Religion (Hindu or Sikh) was included as a covariate. VCE (variance component estimation, originally developed for agricultural data), uses multivariate analysis, incorporating partial correlations between the traits; this provides improved estimates of heritability compared with univariate and bivariate methods. Details of the multivariate models used in VCE are in the ESM methods.

Variance components were estimated incorporating all eight traits: systolic BP (SBP), BMI, WHR, $\log _{e}$ glucose, $\log _{e}$ HDL-cholesterol, $\log _{e} \mathrm{TG}, \mathrm{HbA}_{1 \mathrm{c}}$ and $\log _{e}$ HOMA-IR simultaneously in a linear mixed model. We could not include both BP traits (SBP and diastolic BP [DBP]) in the model because of a high genetic correlation between them $\left(r_{A}=96 \%\right.$; data not shown), which causes computational problems. We decided to include SBP. The model in univariate form is given by $y=X b+a+c+e$, where $y$ is a vector of observed trait values, and $a, c$ and $e$ are vectors of random effects: additive genetic $(a)$, common environment $(c)$ and residual $(e)$. The term $X b$ represents fixed covariate effects. The correlation structure of $a$ is given by kinships derived from each pedigree. Mother was used as a proxy for the common environment, i.e. individuals with the same mother are assumed to have shared the same early childhood environment [5]. The residual effects $e$ are assumed to be uncorrelated.

Heritability $\left(h^{2}\right)$ is the proportion of phenotypic variation in a population that is attributable to additive genetic variation, calculated as the ratio of the genetic variance under an additive model $\left(V_{A}\right)$ to the total phenotypic variance $\left(V_{P}\right)$ [6]. The common environmental effect $\left(c^{2}\right)$ is the proportion of phenotypic variance that is attributable to shared environment within a family, calculated as the ratio of the within-family common environment component $\left(V_{C}\right)$ to $V_{P}$. Genetic correlations $\left(r_{A}\right)$ and common environmental correlations $\left(r_{C}\right)$ are measures of the extent to which the resemblance between relatives can be attributed to shared genetic or environmental factors, respectively.

To minimise potential bias in measured phenotypes introduced by treatments effects, our first analysis excluded observations for individuals on treatment affecting the phenotype. Observations for glucose, HOMA-IR and 
$\mathrm{HbA}_{1 \mathrm{c}}$ were excluded for individuals on type 2 diabetes treatment; observations for SBP, HDL-cholesterol and TG were excluded for individuals on CHD treatment; observations for SBP were excluded for treatment for hypertension; and HDL-cholesterol and TG observations were excluded for individuals on lipid-lowering medication. Data were separately analysed including all individuals, with adjustment $(0,1)$ for treatment of type 2 diabetes, hypertension, dyslipidaemia or CHD ('all participants').

\section{Results}

Characteristics of participants Characteristics of participants are summarised in Table 1. Among all participants, median age was 39.4 years with $52 \%$ male; overall $22 \%$ had CHD, type 2 diabetes or were on treatment for hypertension or dyslipidaemia.

Phenotype information was available for 1,634 of the 2,188 relatives in the families included. On average, there were 12 people (range 3-48), and three generations (range 2-5) per family. A summary of the number of relative pairs for full pedigrees and the phenotyped subsets is in ESM Table 2.

Heritabilities and genetic correlations Table 2 presents heritabilities and common environmental effects for the untreated group, and for all participants. All heritability estimates were statistically significant except for $\log _{e}$ glucose in the treated group. Heritability estimates (SE) for the untreated group were: BMI $0.31(0.04)$, WHR 0.27 (0.04), SBP 0.29 (0.03), TG 0.40 (0.04), HDL-cholesterol
0.53 (0.04), glucose 0.37 (0.03), HOMA-IR $0.22(0.04)$ and $\mathrm{HbA}_{1 \mathrm{c}} 0.60$ (0.04). Heritability estimates were lower for all phenotypes when analysed among all participants. Common environmental effects were low for all traits, and for the untreated group were only significant for SBP, BMI and $\log _{e}$ HOMA-IR.

Genetic correlations are shown in Table 3. In the untreated group, a correlation $>|0.5|$ was found between BMI and $\log _{e}$ HOMA-IR, and correlations $>|0.3|$ were found for: WHR and both SBP and BMI; $\log _{e}$ HOMA-IR and $\log _{e}$ glucose; and between $\log _{e}$ HDL-cholesterol and each of $\log _{e} \mathrm{TG}$ and $\mathrm{HbA}_{1 \mathrm{c}}$. All these, and several correlations of smaller magnitude, were statistically significant.

\section{Discussion}

Family and twin studies indicate that IR, and its related metabolic phenotypes are heritable in Europeans and other populations (ESM Tables 3 and 4). Ours is the first largescale study showing that IR and component phenotypes are highly heritable amongst Asian Indians. Our findings are consistent with the view that genetic factors contribute to the high prevalence of IR and related metabolic disturbances in this population.

The heritabilities reported here provide strong support for efforts to discover new genetic loci underlying IR and related metabolic disturbances in Asian Indians. Heritabilities refer to a particular population, hence comparison of estimates across populations is not straightforward, as implied by the wide range of estimates (ESM Tables 3
Table 1 Phenotypic characteristics of participants after removing outliers

Data are presented as median (inter-quartile range) or \%

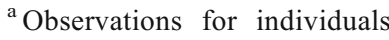
not on BP-, lipid- or glucoselowering drug therapy

${ }^{\mathrm{b}}$ Number of observations

\begin{tabular}{|c|c|c|c|c|}
\hline \multirow[t]{2}{*}{ Characteristic } & \multicolumn{2}{|l|}{ All } & \multicolumn{2}{|c|}{ No drug therapy ${ }^{a}$} \\
\hline & $n^{\mathrm{b}}$ & Value & $n^{\mathrm{b}}$ & Value \\
\hline Age (years) & 1,634 & $39.4(25.9-50.5)$ & - & - \\
\hline Sex (male) $(\%)$ & 1,634 & 52 & & \\
\hline CHD (\%) & 1,634 & 16 & - & - \\
\hline Hypertension on treatment $(\%)$ & 1,634 & 16 & - & - \\
\hline $\mathrm{SBP}(\mathrm{mmHg})$ & 1,622 & $121(110-133)$ & 1,248 & $119(109-129)$ \\
\hline DBP (mmHg) & 1,622 & $77(69-83)$ & 1,248 & $74(68-81)$ \\
\hline BMI $\left(\mathrm{kg} / \mathrm{m}^{2}\right)$ & 1,624 & $25.8(22.9-28.7)$ & - & - \\
\hline WHR & 1,623 & $0.87(0.79-0.94)$ & - & - \\
\hline Lipid-lowering treatment $(\%)$ & 1,634 & 8 & - & - \\
\hline TG (mmol/l) & 1,600 & $1.32(0.92-1.91)$ & 1,338 & $1.23(0.88-1.82)$ \\
\hline HDL-cholesterol (mmol/l) & 1,601 & $1.22(1.04-1.48)$ & 1,339 & $1.25(1.07-1.50)$ \\
\hline Type 2 diabetes on treatment (\%) & 1,634 & 9 & - & - \\
\hline Glucose (mmol/l) & 1,555 & $5.1(4.7-5.5)$ & 1,457 & $5.1(4.7-5.4)$ \\
\hline $\mathrm{HbA}_{1 \mathrm{c}}(\%)$ & 1,107 & $4.9(4.6-5.2)$ & 1,019 & $4.8(4.6-5.1)$ \\
\hline HOMA-IR & 1,538 & $1.9(1.2-3.2)$ & 1,404 & $1.8(1.2-2.8)$ \\
\hline
\end{tabular}


Table 2 Ratios of the estimated variance components for all eight traits from all participants and the untreated group

\begin{tabular}{|c|c|c|c|c|}
\hline \multirow[t]{2}{*}{ Phenotype } & \multicolumn{2}{|l|}{ Untreated group } & \multicolumn{2}{|l|}{ All participants ${ }^{\mathrm{b}}$} \\
\hline & Heritabilities, $h^{2}$ (SE) & Common environment, $c^{2}$ (SE) & Heritabilities, $h^{2}$ (SE) & Common environment, $c^{2}$ (SE) \\
\hline SBP & $0.29(0.03)^{* *}$ & $0.11(0.03)^{* *}$ & $0.18(0.03)^{* *}$ & $0.12(0.02)^{* *}$ \\
\hline BMI & $0.31(0.04)^{* *}$ & $0.13(0.03)^{* *}$ & $0.30(0.03)^{* *}$ & $0.13(0.02)^{* *}$ \\
\hline WHR & $0.27(0.04)^{* *}$ & $0.04(0.02)$ & $0.24(0.03)^{* *}$ & $0.09(0.02)^{* *}$ \\
\hline $\log _{e}$ glucose & $0.37(0.03)^{* *}$ & $0.04(0.02)$ & $0.07(0.03)$ & $0.07(0.02)^{*}$ \\
\hline $\log _{e}$ HDL-C & $0.53(0.04)^{* *}$ & $0.03(0.02)$ & $0.45(0.03)^{* *}$ & $0.05(0.01)^{* *}$ \\
\hline $\log _{e} \mathrm{TG}$ & $0.40(0.04)^{* *}$ & $0.07(0.04)$ & $0.09(0.02)^{* *}$ & $0.03(0.01)$ \\
\hline $\mathrm{HbA}_{1 \mathrm{c}}$ & $0.60(0.04)^{* *}$ & $0.03(0.02)$ & $0.16(0.03)^{* *}$ & $0.15(0.04)^{* *}$ \\
\hline $\log _{e}$ HOMA-IR & $0.22(0.04)^{* *}$ & $0.10(0.03)^{*}$ & $0.19(0.03)^{* *}$ & $0.09(0.02)^{* *}$ \\
\hline
\end{tabular}

${ }^{\text {a }}$ Untreated group: individuals with type 2 diabetes, CHD, hypertension and lipid-lowering drugs excluded

${ }^{\mathrm{b}}$ All participants: all individuals included, with adjustment for treatment effects

${ }^{*} p<0.01, * * p<0.001$, Wald test

HDL-C, HDL-cholesterol

and 4). However, in this study, we found a significant contribution of genetic factors for this population, especially for HDL-cholesterol, $\mathrm{TG}$ and $\mathrm{HbA}_{1 \mathrm{c}}$.

It has been suggested that genes influencing IR have pleiotropic effects on metabolic syndrome-related traits. We found moderate and high genetic correlations between BMI, WHR and impaired HOMA-IR. This implies that genes underlying an increase in general and central adiposity may also influence IR in this population. Support for this view comes from recent studies that show that variants near the $M C 4 R$ gene are associated with both central adiposity and IR in Asian Indians [7].

Like other studies of heritability, our study has limitations. Heritability estimates reflect the ratio of genetic and total variance, and can therefore be influenced by population variance, disease and treatment effects. We therefore

Table 3 Genetic correlations between the traits from all participants and the untreated group

\begin{tabular}{|c|c|c|c|c|c|c|c|}
\hline Phenotype & BMI & WHR & $\begin{array}{l}\log _{e} \\
\text { glucose }\end{array}$ & $\begin{array}{l}\log _{e} \text { HDL- } \\
\text { cholesterol }\end{array}$ & $\log _{e} \mathrm{TG}$ & $\mathrm{HbA}_{1 \mathrm{c}}$ & $\begin{array}{l}\log _{e} \text { HOMA- } \\
\text { IR }\end{array}$ \\
\hline \multicolumn{8}{|l|}{ Untreated group $^{\mathrm{a}}$} \\
\hline SBP & $0.01(0.09)$ & $0.32(0.09)^{* *}$ & $-0.16(0.09)$ & $0.01(0.07)$ & $0.16(0.08)$ & $0.11(0.07)$ & $-0.18(0.12)$ \\
\hline BMI & & $0.44(0.06)^{* *}$ & $0.09(0.07)$ & $0.09(0.07)$ & $0.05(0.08)$ & $0.25(0.05)^{* *}$ & $0.57(0.07)^{* *}$ \\
\hline WHR & & & $-0.22(0.10)$ & $0.02(0.08)$ & $0.27(0.09)^{*}$ & $0.28(0.06)^{* *}$ & $0.24(0.09)^{*}$ \\
\hline $\log _{e}$ glucose & & & & $-0.28(0.05)^{* *}$ & $0.01(0.06)$ & $0.19(0.06)^{* *}$ & $0.35(0.07)^{* *}$ \\
\hline $\log _{e}$ HDL-cholesterol & & & & & $-0.34(0.06)^{* *}$ & $-0.38(0.07)^{* *}$ & $-0.13(0.09)$ \\
\hline $\log _{e} \mathrm{TG}$ & & & & & & $0.25(0.06)$ & $0.18(0.10)$ \\
\hline $\mathrm{HbA}_{1 \mathrm{c}}$ & & & & & & & $0.16(0.07)$ \\
\hline \multicolumn{8}{|l|}{ All participants ${ }^{\mathrm{b}}$} \\
\hline SBP & $0.12(0.07)$ & $0.34(0.07)^{* *}$ & $-0.09(0.13)$ & $-0.01(0.05)$ & $0.12(0.14)$ & $0.06(0.10)$ & $-0.15(0.08)$ \\
\hline BMI & & $0.56(0.06)^{* *}$ & $0.08(0.13)$ & $0.15(0.06)$ & $-0.10(0.10)$ & $0.10(0.09)$ & $0.54(0.09)^{* *}$ \\
\hline WHR & & & $-0.11(0.15)$ & $-0.04(0.05)$ & $0.40(0.17)$ & $0.39(0.08)^{* *}$ & $0.23(0.09)^{*}$ \\
\hline $\log _{e}$ glucose & & & & $-0.07(0.09)$ & $0.22(0.16)$ & $-0.15(0.16)$ & $0.32(0.11)^{*}$ \\
\hline $\log _{e}$ HDL-cholesterol & & & & & $-0.50(0.11)^{* *}$ & $-0.41(0.08)^{* *}$ & $-0.19(0.07)^{*}$ \\
\hline $\log _{e} \mathrm{TG}$ & & & & & & $0.15(0.23)$ & $-0.12(0.14)$ \\
\hline $\mathrm{HbA}_{1 \mathrm{c}}$ & & & & & & & $-0.30(0.18)$ \\
\hline
\end{tabular}

Values are $r_{A}(\mathrm{SE})$

${ }^{\text {a }}$ Untreated group: individuals with type 2 diabetes, CHD, hypertension and lipid-lowering drugs excluded

${ }^{\mathrm{b}}$ All participants: all individuals included, with adjustment for treatment effects

${ }^{*} p<0.01, * * p<0.001$, Wald test 
estimated heritability both with and without participants on treatment for diabetes, hypertension, raised lipids or CHD; estimates were consistently higher without these participants. Our use of a multivariate model to analyse all traits simultaneously allows more precise estimation, and taking a shared environment into account should avoid an upward bias that can affect studies ignoring these effects.

In conclusion, our findings of moderate and high heritabilities for, and significant genetic correlations between, IR component phenotypes in Asian Indian CHD families suggest that genetic effects contribute substantially to IR in this population. Our results support the view that common genetic mechanisms underlie IR and related metabolic disturbances amongst Asian Indians.

Acknowledgements The authors would like to thank all the members of the families that participated in the study. D. Zabaneh is funded by the British Heart Foundation. Part of this work appeared as an abstract in the 16th Annual Meeting of the International Genetic Epidemiology Society 31:651-651 (September 2007).
Duality of interest The authors declare that there is no duality of interest associated with this manuscript

\section{References}

1. Defronzo RA, Ferrannini E (1991) Insulin resistance: a multifaceted syndrome responsible for NIDDM, obesity, hypertension, dyslipidemia, and atherosclerotic cardiovascular disease. Diabetes Care 14:173-194

2. Hill JO, Wyatt HR, Reed GW, Peters JC (2003) Obesity and the environment: where do we go from here? Science 299:853-855

3. Lopez AD, Murray CCJL (1998) The global burden of disease, 1990-2020. Nat Med 4:1241-1243

4. Neumaier A, Groeneveld E (1998) Restricted maximum likelihood estimation of covariances in sparse linear models. Genet Sel Evol 30:3-26

5. Barroso I (2005) Genetics of type 2 diabetes. Diabet Med 22:517535

6. Falconer DS, Mackay TFC (1996) Introduction to quantitative genetics, 4th edn. Longman, Harlow

7. Chambers JC, Elliott P, Zabaneh D et al (2008) Common genetic variation near MC4R is associated with waist circumference and insulin resistance. Nat Genet 40:716-718 